\title{
Uniqueness of meromorphic functions when two linear differential polynomials share the same 1-points
}

\author{
by InDRaJit Lahiri (Calcutta and Kalyani)
}

\begin{abstract}
We prove a uniqueness theorem for meromorphic functions involving linear differential polynomials generated by them. As consequences of the main result we improve some previous results.
\end{abstract}

1. Introduction. Let $f$ and $g$ be two nonconstant meromorphic functions defined in the open complex plane $\mathbb{C}$. If for $a \in \mathbb{C} \cup \infty, f-a$ and $g-a$ have the same set of zeros with the same multiplicities, we say that $f$ and $g$ share the value a $C M$ (counting multiplicities), and if we do not consider the multiplicities, $f$ and $g$ are said to share the value a IM (ignoring multiplicities). It is assumed that the reader is familiar with the standard notations and definitions of value distribution theory (cf. [3]).

M. Ozawa [6] proved the following result:

THEOREM A [6]. If two nonconstant entire functions $f, g$ share the value $1 C M$ with $\delta(0 ; f)>0$ and 0 being lacunary for $g$ then either $f \equiv g$ or $f g \equiv 1$.

Improving the above result $\mathrm{H}$. X. Yi [10] proved the following:

THEOREM B [10]. Let $f$ and $g$ be two nonconstant meromorphic functions satisfying $\delta(\infty ; f)=\delta(\infty ; g)=1$. If $f, g$ share the value $1 C M$ and $\delta(0 ; f)+$ $\delta(0 ; g)>1$ then either $f \equiv g$ or $f g \equiv 1$.

In [9] C. C. Yang asked: What can be said if two nonconstant entire functions $f$ and $g$ share the value $0 \mathrm{CM}$ and their first derivatives share the value $1 \mathrm{CM}$ ?

As an attempt to solve this question K. Shibazaki [7] proved the following:

1991 Mathematics Subject Classification: Primary 30D35.

Key words and phrases: uniqueness, sharing values, differential polynomial. 
Theorem C [7]. Let $f$ and $g$ be two entire functions of finite order. If $f^{\prime}$ and $g^{\prime}$ share the value $1 C M$ with $\delta(0 ; f)>0$ and 0 being lacunary for $g$ then either $f \equiv g$ or $f^{\prime} g^{\prime} \equiv 1$.

Improving Theorem C, H. X. Yi [13] obtained the following result:

Theorem D [13]. Let $f$ and $g$ be two entire functions such that $f^{(n)}$ and $g^{(n)}$ share the value $1 C M$. If $\delta(0 ; f)+\delta(0 ; g)>1$ then either $f \equiv g$ or $f^{(n)} g^{(n)} \equiv 1$.

Considering meromorphic functions H. X. Yi and and C. C. Yang [15] improved Theorem $\mathrm{C}$ as follows:

TheOrem E [15]. Let $f$ and $g$ be two meromorphic functions satisfying $\delta(\infty ; f)=\delta(\infty ; g)=1$. If $f^{\prime}$ and $g^{\prime}$ share the value $1 C M$ with $\delta(0 ; f)+$ $\delta(0 ; g)>1$ then either $f \equiv g$ or $f^{\prime} g^{\prime} \equiv 1$.

In [15] it is asked whether it is possible to replace the first derivatives $f^{\prime}, g^{\prime}$ in Theorem E by the $n$th derivatives $f^{(n)}$ and $g^{(n)}$.

In this direction the following two theorems can be noted.

TheOREm $\mathrm{F}$ [13]. Let $f$ and $g$ be two meromorphic functions sharing the value $\infty$ CM. If $f^{(n)}$ and $g^{(n)}$ share the value $1 C M$ with $\delta(0 ; f)+\delta(0 ; g)+$ $(n+2) \Theta(\infty ; f)>n+3$ then either $f \equiv g$ or $f^{(n)} g^{(n)} \equiv 1$.

Theorem G [16]. Let $f$ and $g$ be two meromorphic functions such that $\Theta(\infty ; f)=\Theta(\infty ; g)=1$. If $f^{(n)}$ and $g^{(n)}$ share the value $1 C M$ and $\delta(0 ; f)+$ $\delta(0 ; g)>1$ then either $f \equiv g$ or $f^{(n)} g^{(n)} \equiv 1$.

So it is not irrelevant to ask: What can be said if two linear differential polynomials generated by two meromorphic functions $f$ and $g$ share the value $1 \mathrm{CM}$ ?

In the paper we answer this question. Also as a consequence of the main theorem we prove a result which improves Theorem $\mathrm{G}$ and so some previous results.

2. Definitions and notations. In this section we present some necessary notations and definitions.

Notation 1 . We denote by $\Psi(D)$ a linear differential operator with constant coefficients of the form $\Psi(D)=\sum_{i=1}^{p} \alpha_{i} D^{i}$, where $D \equiv d / d z$.

DeFinition 1. For a meromorphic function $f$ and a positive integer $k$, $N_{k}(r, a ; f)$ denotes the counting function of $a$-points of $f$ where an $a$-point with multiplicity $m$ is counted $m$ times if $m \leq k$ and $k$ times if $m>k$. 
Definition 2 (cf. [1]). For a meromorphic function $f$ we put

$$
\begin{aligned}
T_{0}(r, f) & =\int_{1}^{r} \frac{T(t, f)}{t} d t \\
N_{0}(r, a ; f) & =\int_{1}^{r} \frac{N(t, a ; f)}{t} d t, \quad N_{k}^{0}(r, a ; f)=\int_{1}^{r} \frac{N_{k}(t, a ; f)}{t} d t, \\
m_{0}(r, f) & =\int_{1}^{r} \frac{m(t, f)}{t} d t, \quad S_{0}(r, f)=\int_{1}^{r} \frac{S(t, f)}{t} d t \quad \text { etc. }
\end{aligned}
$$

DeFinition 3. If $f$ is a meromorphic function, then

$$
\delta_{k}(a ; f)=1-\limsup _{r \rightarrow \infty} \frac{N_{k}(r, a ; f)}{T(r, f)} .
$$

Clearly $0 \leq \delta(a ; f) \leq \delta_{k}(a ; f) \leq \delta_{k-1}(a ; f) \leq \ldots \leq \delta_{2}(a ; f) \leq \delta_{1}(a ; f)=$ $\Theta(a ; f) \leq 1$.

Definition 4 (cf. [8]). For a meromorphic function $f$ we put

$$
\begin{array}{ll}
\delta_{0}(a ; f)=1-\limsup _{r \rightarrow \infty} \frac{N_{0}(r, a ; f)}{T_{0}(r, f)}, \quad \Theta_{0}(a ; f)=1-\limsup _{r \rightarrow \infty} \frac{\bar{N}_{0}(r, a ; f)}{T_{0}(r, f)}, \\
\delta_{k}^{0}(a ; f)=1-\limsup _{r \rightarrow \infty} \frac{N_{k}^{0}(r, a ; f)}{T_{0}(r, f)} \quad \text { where } a \in \mathbb{C} \cup \infty .
\end{array}
$$

3. Lemmas. In this section we discuss some lemmas which will be required in the sequel.

Lemma 1 [1]. For meromorphic $f$,

$$
\lim _{r \rightarrow \infty} \frac{S_{0}(r, f)}{T_{0}(r, f)}=0
$$

through all values of $r$.

Lemma 2. Let $f$ be a meromorphic function and $a \in \mathbb{C} \cup \infty$. Then $\delta(a ; f) \leq \delta_{0}(a ; f), \Theta(a ; f) \leq \Theta_{0}(a ; f)$ and $\delta_{k}(a ; f) \leq \delta_{k}^{0}(a ; f)$.

This lemma can be proved along the lines of [7, Proposition 6].

LEMMA 3. Let $f_{1}, f_{2}$ be nonconstant meromorphic functions such that $a f_{1}+b f_{2} \equiv 1$, where $a, b$ are nonzero constants. Then

$$
T_{0}\left(r, f_{1}\right) \leq \bar{N}_{0}\left(r, 0 ; f_{1}\right)+\bar{N}_{0}\left(r, 0 ; f_{2}\right)+\bar{N}_{0}\left(r, \infty ; f_{1}\right)+S_{0}\left(r, f_{1}\right) .
$$

Proof. By the second fundamental theorem we get

$$
\begin{aligned}
T\left(r, f_{1}\right) & \leq \bar{N}\left(r, 0 ; f_{1}\right)+\bar{N}\left(r, a^{-1} ; f_{1}\right)+\bar{N}\left(r, \infty ; f_{2}\right)+S\left(r, f_{1}\right) \\
& =\bar{N}\left(r, 0 ; f_{1}\right)+\bar{N}\left(r, 0, f_{2}\right)+\bar{N}\left(r, \infty ; f_{1}\right)+S\left(r, f_{1}\right) .
\end{aligned}
$$

From this inequality the lemma follows on integration. 
Lemma 4 [4]. For a meromorphic function $f$ and any $a \in \mathbb{C}$,

$$
N(r, 0 ; \Psi(D) f \mid f=a, \geq p) \geq N\left(r, 0 ; f^{(p)} \mid f=a, \geq p\right)+S(r, f),
$$

where $N(r, b ; g \mid f=c, \geq k)$ is the counting function of those b-points of $g$, counted with proper multiplicities, which are the c-points of $f$ with multiplicities not less than $k$.

LEMma 5. Let $f$ be a meromorphic function. Then

$$
\begin{aligned}
& \liminf _{r \rightarrow \infty} \frac{T_{0}(r, \Psi(D) f)}{T_{0}(r, f)} \geq \sum_{a \neq \infty} \delta_{p}^{0}(a ; f), \\
& \delta_{0}(0 ; \Psi(D) f) \geq \frac{\sum_{a \neq \infty} \delta_{0}(a ; f)}{1+p\left(1-\Theta_{0}(\infty ; f)\right)} .
\end{aligned}
$$

Proof. For distinct finite complex numbers $a_{1}, \ldots, a_{n}$ we put

$$
A=\sum_{i=1}^{n} \frac{1}{f-a_{i}} .
$$

Then by $[3$, inequality 2.1, p. 33$]$ we get

$$
\begin{aligned}
\sum_{i=1}^{n} m\left(r, a_{i} ; f\right) & \leq m(r, A)+O(1) \\
& \leq m(r, 0 ; \Psi(D) f)+m(r, A \Psi(D) f) \\
& \leq m(r, 0 ; \Psi(D) f)+\sum_{i=1}^{n} m\left(r, \frac{\Psi(D) f}{f-a_{i}}\right) \\
& =m(r, 0 ; \Psi(D) f)+\sum_{i=1}^{n} m\left(r, \frac{\Psi(D)\left(f-a_{i}\right)}{f-a_{i}}\right) \\
& =m(r, 0 ; \Psi(D) f)+S(r, f),
\end{aligned}
$$

by the Milloux theorem [3, p. 55], i.e.,

$$
\begin{aligned}
\text { (1) } n T(r, f) \leq & T(r, \Psi(D) f)+\sum_{i=1}^{n} N\left(r, a_{i} ; f\right)-N(r, 0 ; \Psi(D) f)+S(r, f) \\
\leq & T(r, \Psi(D) f) \\
& +\sum_{i=1}^{n}\left\{N\left(r, a_{i} ; f\right)-N\left(r, 0 ; \Psi(D) f \mid f=a_{i}, \geq p\right)\right\} \\
& +S(r, f) .
\end{aligned}
$$


So by Lemma 4 we get

$$
\begin{aligned}
n T(r, f) \leq & T(r, \Psi(D) f)+\sum_{i=1}^{n}\left\{N\left(r, a_{i} ; f\right)-N\left(r, 0 ; f^{(p)} \mid f=a_{i}, \geq p\right)\right\} \\
& +S(r, f) \\
\leq & T(r, \Psi(D) f)+\sum_{i=1}^{n} N_{p}\left(r, a_{i} ; f\right)+S(r, f) .
\end{aligned}
$$

This gives on integration

$$
n T_{0}(r, f) \leq T_{0}(r, \Psi(D) f)+\sum_{i=1}^{n} N_{p}^{0}\left(r, a_{i} ; f\right)+S_{0}(r, f) .
$$

Hence by Lemma 1 we get

$$
\liminf _{r \rightarrow \infty} \frac{T_{0}(r, \Psi(D) f)}{T_{0}(r, f)} \geq \sum_{i=1}^{n} \delta_{p}^{0}\left(a_{i} ; f\right) .
$$

Since $n$ is arbitrary, it follows that

$$
\liminf _{r \rightarrow \infty} \frac{T_{0}(r, \Psi(D) f)}{T_{0}(r, f)} \geq \sum_{a \neq \infty} \delta_{p}^{0}(a ; f) .
$$

Again by the Milloux theorem,

$$
\begin{aligned}
T(r, \Psi(D) f) \leq & m\left(r, \frac{\Psi(D) f}{f}\right)+m(r, f)+N(r, f) \\
& +p \bar{N}(r, f)+O(1) \\
= & T(r, f)+p \bar{N}(r, f)+S(r, f) .
\end{aligned}
$$

This gives on integration

$$
T_{0}(r, \Psi(D) f) \leq T_{0}(r, f)+p \bar{N}_{0}(r, f)+S_{0}(r, f) .
$$

Also from (1) we get by integration

$$
\begin{aligned}
n T_{0}(r, f) \leq & T_{0}(r, \Psi(D) f)+\sum_{i=1}^{n} N_{0}\left(r, a_{i} ; f\right) \\
& -N_{0}(r, 0 ; \Psi(D) f)+S_{0}(r, f) .
\end{aligned}
$$

So by (2) we obtain

$$
\begin{aligned}
n \leq & \left(1-\frac{N_{0}(r, 0 ; \Psi(D) f)}{T_{0}(r, \Psi(D) f)}\right) \cdot \frac{T_{0}(r, f)+p \bar{N}_{0}(r, f)+S_{0}(r, f)}{T_{0}(r, f)} \\
& +\sum_{i=1}^{n} \frac{N_{0}\left(r, a_{i} ; f\right)}{T_{0}(r, f)}+\frac{S_{0}(r, f)}{T_{0}(r, f)} .
\end{aligned}
$$


In view of Lemma 1 this gives

$$
\left.\sum_{i=1}^{n} \delta_{0}\left(a_{i} ; f\right) \leq \delta_{0}(0 ; \Psi(D) f)\left\{1-\Theta_{0}(\infty ; f)\right)\right\},
$$

from which (ii) follows because $n$ is arbitrary. This proves the lemma.

LEMma 6 [11]. Let $f_{1}, f_{2}, f_{3}$ be nonconstant meromorphic functions satisfying $f_{1}+f_{2}+f_{3} \equiv 1$. If $f_{1}, f_{2}, f_{3}$ are linearly independent then $g_{1}=$ $-f_{2} / f_{3}, g_{2}=1 / f_{3}$ and $g_{3}=-f_{1} / f_{3}$ are also linearly independent.

Lemma 7. Let $f_{1}, f_{2}, f_{3}$ be three linearly independent meromorphic functions such that $f_{1}+f_{2}+f_{3} \equiv 1$. Then

$$
\begin{aligned}
T_{0}\left(r, f_{1}\right) \leq & \sum_{j=1}^{3} N_{2}^{0}\left(r, 0 ; f_{j}\right)+\max _{1 \leq i \neq j \leq 3}\left\{N_{2}^{0}\left(r, \infty ; f_{i}\right)+\bar{N}_{0}\left(r, \infty ; f_{j}\right)\right\} \\
& +S_{0}(r),
\end{aligned}
$$

where $S_{0}(r)=\sum_{j=1}^{3} S_{0}\left(r, f_{j}\right)$.

Proof. We prove under the hypotheses of the lemma the following inequality which on integration proves the lemma:

$$
\begin{aligned}
T\left(r, f_{1}\right) \leq & \sum_{j=1}^{3} N_{2}\left(r, 0 ; f_{j}\right)+\max _{1 \leq i \neq j \leq 3}\left\{N_{2}\left(r, \infty ; f_{i}\right)+\bar{N}\left(r, \infty ; f_{j}\right)\right\} \\
& +\sum_{j=1}^{3} S\left(r, f_{j}\right) .
\end{aligned}
$$

From the proof of a generalisation of Borel's theorem by Nevanlinna (cf. $[2$, p. 70]) we get

$$
\begin{aligned}
T\left(r, f_{1}\right) \leq & \sum_{j=1}^{3} N\left(r, 0 ; f_{j}\right)-N(r, 0 ; \Delta)+N(r, \Delta) \\
& -N\left(r, f_{2}\right)-N\left(r, f_{3}\right)+S(r),
\end{aligned}
$$

where $\Delta$ is the wronskian determinant of $f_{1}, f_{2}, f_{3}$ and $S(r)=\sum_{j=1}^{3} S\left(r, f_{j}\right)$.

Now we need the following notations from [5]: for $z \in \mathbb{C}$ and $b \in \mathbb{C} \cup\{\infty\}$ we put

$$
\begin{aligned}
& \mu_{f}^{b}(z)= \begin{cases}m & \text { if } z \text { is a } b \text {-point of } f \text { with multiplicity } m \geq 1, \\
0 & \text { if } z \text { is not a } b \text {-point of } f,\end{cases} \\
& \bar{\mu}_{f}^{b}(z)= \begin{cases}1 & \text { if } z \text { is a } b \text {-point of } f \text { with multiplicity } \geq 1, \\
0 & \text { if } z \text { is not a } b \text {-point of } f,\end{cases} \\
& \nu_{f}^{b}(z)= \begin{cases}2 & \text { if } z \text { is a } b \text {-point of } f \text { with multiplicity } m>2, \\
m & \text { if } z \text { is a } b \text {-point of } f \text { with multiplicity } m \leq 2 .\end{cases}
\end{aligned}
$$


Also we put

$$
\mu(z)=\sum_{j=1}^{3} \mu_{f_{j}}^{0}(z)-\mu_{\Delta}^{0}(z)+\mu_{\Delta}^{\infty}(z)-\mu_{f_{2}}^{\infty}(z)-\mu_{f_{3}}^{\infty}(z)
$$

and

$$
\mu^{*}(z)=\sum_{j=1}^{3} \nu_{f_{j}}^{0}(z)+\max _{1 \leq i \neq j \leq 3}\left\{\nu_{f_{i}}^{\infty}(z)+\bar{\mu}_{f_{j}}^{\infty}(z)\right\} .
$$

Now (3) will follow from (4) if we can prove that for any $z \in \mathbb{C}, \mu(z) \leq \mu^{*}(z)$.

We consider the following cases.

CASE 1 . Let $z$ be not a pole of any $f_{i}(i=1,2,3)$. Since any zero of $f_{i}$ with multiplicity $m>2$ is a zero of $\Delta$ with multiplicity at least $m-2$, it follows that $\mu(z) \leq \mu^{*}(z)$.

CASE 2. Let $z$ be a pole of at least one of $f_{i}(i=1,2,3)$. So the following subcases come up for consideration.

SUBCASE 2.1. Let $z$ be a zero of $f_{1}$ with multiplicity $m>2$ and a pole of $f_{2}, f_{3}$ with multiplicity $k \geq 1$. Then $z$ is a pole of $\Delta$ with multiplicity $k-m+3$ provided $k-m+3>0$ and otherwise $z$ is a zero of $\Delta$ with multiplicity $m-k-3$. Hence $\mu(z)=3-k$ and $\mu^{*}(z) \geq 3$. So $\mu(z) \leq \mu^{*}(z)$.

Let $z$ be a zero of $f_{1}$ with multiplicity $m \leq 2$ and a pole of $f_{2}, f_{3}$ with multiplicity $k \geq 1$. Then $z$ is a pole $\Delta$ with multiplicity not exceeding $k+2$. Hence $\mu(z) \leq m+k+2-k-k \leq 4-k$ and $\mu^{*}(z) \geq 3$. So $\mu(z) \leq \mu^{*}(z)$.

SUBCASE 2.2. Let $z$ be a zero of $f_{2}$ with multiplicity $m>2$ and a pole of $f_{1}, f_{3}$ with multiplicity $k \geq 1$. Then $z$ is a pole of $\Delta$ with multiplicity $k-m+3$ provided $k-m+3>0$ and otherwise $z$ is a zero of $\Delta$ with multiplicity $m-k-3$. Hence $\mu(z)=3$ and $\mu^{*}(z) \geq 3$. So $\mu(z) \leq \mu^{*}(z)$.

Let $z$ be a zero of $f_{2}$ with multiplicity $m \leq 2$ and a pole of $f_{1}, f_{3}$ with multiplicity $k \geq 1$. Then $z$ is a pole of $\Delta$ with multiplicity not exceeding $k+2$. Hence $\mu(z) \leq m+k+2-k=m+2$ and $\mu^{*}(z) \geq m+2$. So $\mu(z) \leq \mu^{*}(z)$.

SubCASE 2.3. Let $z$ be a zero of $f_{3}$ with multiplicity $m \geq 1$ and a pole of $f_{1}, f_{2}$ with multiplicity $k \geq 1$. Then as in Subcase 2.2 we can prove that $\mu(z) \leq \mu^{*}(z)$.

SubCASE 2.4. Let $z$ be neither a zero nor a pole of $f_{1}$. Since $f_{2}+f_{3}=$ $1-f_{1}$, it follows that $z$ is not a pole of $f_{2}+f_{3}$. Since $z$ is a pole of at least one of $f_{i}(i=1,2,3)$, it follows that $z$ is a pole of $f_{2}$ and $f_{3}$ with the same multiplicity $m$, say (because the singularities of $f_{2}$ and $f_{3}$ at $z$ cancel each other). Then $z$ is a pole of $\Delta$ with multiplicity not exceeding $m+2$. Hence $\mu(z) \leq m+2-m-m \leq 2$ and $\mu^{*}(z) \geq 2$. So $\mu(z) \leq \mu^{*}(z)$.

SUBCASE 2.5. Let $z$ be a pole of $f_{1}, f_{2}$ with multiplicity $m \geq 1$ and a pole of $f_{3}$ with multiplicity $q(1 \leq q<m)$. Then $z$ is a pole of $\Delta$ with 
multiplicity not exceeding $m+q+3$. Hence $\mu(z) \leq m+q+3-m-q=3$ and $\mu^{*}(z)=2+1=3$. So $\mu(z) \leq \mu^{*}(z)$.

SUBCASE 2.6. Let $z$ be a pole of $f_{1}, f_{2}, f_{3}$ with multiplicity $m \geq 1$. Then there exist two functions $\phi, \psi$ analytic at $z$ and $\phi(z) \neq 0, \psi(z) \neq 0$ such that in some neighbourhood of $z, f_{2}(\omega)=(\omega-z)^{-m} \phi(\omega)$ and $f_{3}(\omega)$ $=(\omega-z)^{-m} \psi(\omega)$. Also $\Delta=f_{2}^{\prime} f_{3}^{\prime \prime}-f_{2}^{\prime \prime} f_{3}^{\prime}$ shows that $z$ is a pole of $\Delta$ with multiplicity not exceeding $2 m+3$ but by actual calculation we see that the coefficient of $(\omega-z)^{-(2 m+3)}$ in $\Delta$ is $m^{2}(m+1) \phi \psi-m^{2}(m+1) \phi \psi \equiv 0$. So $z$ is a pole of $\Delta$ with multiplicity not exceeding $2 m+2$. Hence $\mu(z) \leq$ $2 m+2-m-m=2$ and $\mu^{*}(z) \geq 2$. So $\mu(z) \leq \mu^{*}(z)$.

SUBCASE 2.7. Let $z$ be a pole of $f_{1}, f_{2}$ with multiplicity $m \geq 1$ and neither a zero nor a pole of $f_{3}$. Then $z$ is a pole of $\Delta$ with multiplicity not exceeding $m+2$. Hence $\mu(z) \leq m+2-m=2$ and $\mu^{*}(z) \geq 2$. So $\mu(z) \leq \mu^{*}(z)$.

SUBCASE 2.8. Let $z$ be a pole of $f_{1}$ with multiplicity $m \geq 1$ and a pole of $f_{2}$ with multiplicity $m+q(q \geq 1)$. Then $z$ is also a pole of $f_{3}$ with multiplicity $m+q$ and the terms containing $(w-z)^{-(m+i)}(i=1, \ldots, q)$ of the Laurent expansions of $f_{2}$ and $f_{3}$ about $z$ cancel each other because $f_{2}+f_{3}$ has a pole at $z$ with multiplicity $m$. Also we see that $\Delta$ has a pole at $z$ with multiplicity not exceeding $2 m+q+3$. Hence $\mu(z) \leq 2 m+q+3-m-q-m-q=3-q$ and $\mu^{*}(z)=2+1=3$. So $\mu(z) \leq \mu^{*}(z)$.

Lemma 8. If $\sum_{a \neq \infty} \delta_{p}^{0}(a ; f)>0$ then

$$
\Theta_{0}(\infty ; \Psi(D) f) \geq 1-\frac{1-\Theta_{0}(\infty ; f)}{\sum_{a \neq \infty} \delta_{p}^{0}(a ; f)} .
$$

Pro of. Since $\bar{N}_{0}(r, \Psi(D) f)=\bar{N}_{0}(r, f)$, the lemma follows from Lemma $5(\mathrm{i})$.

Lemma 9 [14]. Let $F$ and $G$ be two nonconstant meromorphic functions such that $F$ and $G$ share $1 C M$. If

$$
\limsup _{r \rightarrow \infty, r \in I} \frac{N_{2}(r, 0 ; F)+N_{2}(r, \infty ; F)+N_{2}(r, 0 ; G)+N_{2}(r, \infty ; G)}{T(r)}<1,
$$

where

$$
T(r)=\max \{T(r, F), T(r, G)\}
$$

and $I$ is a set of $r$ 's $(0<r<\infty)$ of infinite linear measure, then $F \equiv G$ or $F G \equiv 1$.

4. Theorems. In this section we present the main results of the paper.

TheOREM 1. Let $f, g$ be two meromorphic functions such that 
(i) $\Psi(D) f, \Psi(D) g$ are nonconstant and share $1 C M$ and

(ii) $\frac{\sum_{a \neq \infty} \delta(a ; f)}{1+p(1-\Theta(\infty ; f))}+\frac{\sum_{a \neq \infty} \delta(a ; g)}{1+p(1-\Theta(\infty ; g))}$

$$
>1+\frac{4(1-\Theta(\infty ; f))}{\sum_{a \neq \infty} \delta_{p}(a ; f)}+\frac{4(1-\Theta(\infty ; g))}{\sum_{a \neq \infty} \delta_{p}(a ; g)},
$$

where $\sum_{a \neq \infty} \delta_{p}(a ; f)>0$ and $\sum_{a \neq \infty} \delta_{p}(a ; g)>0$. Then either $[\Psi(D) f]$. $[\Psi(D) g] \equiv 1$ or $f-g \equiv s$, where $s$ is a solution of the differential equation $\Psi(D) w=0$.

TheOREM 2. Let $f, g$ be two meromorphic functions of finite order such that

(i) $\Psi(D) f, \Psi(D) g$ are nonconstant and share $1 C M$ and

(ii) $\frac{\sum_{a \neq \infty} \delta(a ; f)}{1+p(1-\Theta(\infty ; f))}+\frac{\sum_{a \neq \infty} \delta(a ; g)}{1+p(1-\Theta(\infty ; g))}$

$$
>1+\frac{2(1-\Theta(\infty ; f))}{\sum_{a \neq \infty} \delta_{p}(a ; f)}+\frac{2(1-\Theta(\infty ; g))}{\sum_{a \neq \infty} \delta_{p}(a ; g)},
$$

where $\sum_{a \neq \infty} \delta_{p}(a ; f)>0$ and $\sum_{a \neq \infty} \delta_{p}(a ; g)>0$. Then either $[\Psi(D) f]$. $[\Psi(D) g] \equiv 1$ or $f-g \equiv s$, where $s$ is a solution of the differential equation $\Psi(D) w=0$.

The following example shows that the theorems are sharp.

EXAMPLE 1. Let $f=\frac{1}{2} e^{z}\left(e^{z}-1\right), g=\frac{1}{2} e^{-z}\left(\frac{1}{2}-\frac{1}{5} e^{-z}\right)$ and $\Psi(D)=D^{2}-$ $3 D$. Then $\sum_{a \neq \infty} \delta(a ; f)=\sum_{a \neq \infty} \delta(a ; g)=1 / 2, \Theta(\infty ; f)=\Theta(\infty ; g)=1$ and $\sum_{a \neq \infty} \delta_{2}(a ; f)>0, \sum_{a \neq \infty} \delta_{2}(a ; g)>0$. Also $\Psi(D) f=e^{z}\left(1-e^{z}\right)$ and $\Psi(D) g=e^{-z}\left(1-e^{-z}\right)$ share $1 \mathrm{CM}$ but neither $[\Psi(D) f] \cdot[\Psi(D) g] \equiv 1$ nor $f-g \equiv c_{1}+c_{2} e^{3 z}$ for any constants $c_{1}$ and $c_{2}$.

Proof of Theorem 1. Let $F=\Psi(D) f$ and $G=\Psi(D) g$. Then in view of Lemmas 2, 5 and 8 the condition (ii) implies

$$
\delta_{0}(0 ; F)+\delta_{0}(0 ; G)+4 \Theta_{0}(\infty ; F)+4 \Theta_{0}(\infty ; G)>9 .
$$

We put

$$
H=\frac{F-1}{G-1} .
$$

Since $F, G$ share $1 \mathrm{CM}$, the poles and zeros of $H$ occur only at the poles of $F$ and $G$ respectively. Also $\bar{N}_{0}(r, \infty ; H) \leq \bar{N}_{0}(r, \infty ; F)$ and $\bar{N}_{0}(r, 0 ; H) \leq$ $\bar{N}_{0}(r, \infty ; G)$.

Let $F_{1}=F, F_{2}=-G H$ and $F_{3}=H$. Then from (6) it follows that

$$
F_{1}+F_{2}+F_{3} \equiv 1 \text {. }
$$


First we suppose that $F_{3}=H \equiv k$, a constant. Then from (7) we get $F-k G=1-k$. If $k \neq 1$, we see that

$$
\frac{1}{1-k} F-\frac{k}{1-k} G \equiv 1 \text {. }
$$

Since $k \neq 0$, from Lemma 3 it follows that

$$
\begin{aligned}
& T_{0}(r, F) \leq N_{0}(r, 0 ; F)+N_{0}(r, 0 ; G)+\bar{N}_{0}(r, \infty ; F)+S_{0}(r, F), \\
& T_{0}(r, G) \leq N_{0}(r, 0 ; F)+N_{0}(r, 0 ; G)+\bar{N}_{0}(r, \infty ; G)+S_{0}(r, G) .
\end{aligned}
$$

So

$$
\begin{aligned}
\max \left\{T_{0}(r, F), T_{0}(r, G)\right\} \leq & N_{0}(r, 0 ; F)+N_{0}(r, 0 ; G)+\bar{N}_{0}(r, \infty ; F) \\
& +\bar{N}_{0}(r, \infty ; G)+o\left(\max \left\{T_{0}(r, F), T_{0}(r, G)\right\}\right) .
\end{aligned}
$$

This gives $\delta_{0}(0 ; F)+\delta_{0}(0 ; G)+\Theta_{0}(\infty ; F)+\Theta_{0}(\infty ; G) \leq 3$ and so from (5) we see that $9<3 \Theta_{0}(\infty ; F)+3 \Theta_{0}(\infty ; G)+3 \leq 9$, a contradiction. So $k=1$ and hence $F \equiv G$. Therefore $\Psi(D)(f-g) \equiv 0$ and so $f-g \equiv s$, where $s=s(z)$ is a solution of $\Psi(D) w=0$.

Similarly if $F_{2} \equiv k$, a constant, we can show that $[\Psi(D) f] \cdot[\Psi(D) g] \equiv 1$.

Now we suppose that $F_{1}, F_{2}$ and $F_{3}$ are nonconstant. If possible, let $F_{1}, F_{2}, F_{3}$ be linearly independent. Then from Lemma 7 we get

(8) $T_{0}(r, F) \leq N_{2}^{0}(r, 0 ; F)+N_{2}^{0}(r, 0 ; G)+2 N_{2}^{0}(r, 0 ; H)$

$$
\begin{aligned}
& +\max _{1 \leq i \neq j \leq 3}\left\{N_{2}^{0}\left(r, \infty ; F_{i}\right)+\bar{N}_{0}\left(r, \infty ; F_{j}\right)\right\}+\sum_{j=1}^{3} S_{0}\left(r, F_{j}\right) \\
\leq & N_{0}(r, 0 ; F)+N_{0}(r, 0 ; G)+4 \bar{N}_{0}(r, \infty ; G) \\
& +\max _{1 \leq 1 \neq j \leq 3}\left\{N_{2}^{0}\left(r, \infty ; F_{i}\right)+\bar{N}_{0}\left(r, \infty ; F_{j}\right)\right\}+\sum_{j=1}^{3} S_{0}\left(r, F_{j}\right) .
\end{aligned}
$$

Now in view of (6) we see that

$$
\sum_{j=1}^{3} S_{0}\left(r, F_{j}\right)=o\left(\max \left\{T_{0}(r, F), T_{0}(r, G)\right\}\right)
$$

and

$$
\begin{aligned}
N_{2}^{0}\left(r, \infty ; F_{1}\right)+\bar{N}_{0}\left(r, \infty ; F_{2}\right) & =N_{2}^{0}(r, \infty ; F)+\bar{N}_{0}(r, \infty ; H(G-1)) \\
& =N_{2}^{0}(r, \infty ; F)+\bar{N}_{0}(r, \infty ; F) \leq 3 \bar{N}_{0}(r, \infty ; F), \\
N_{2}^{0}\left(r, \infty ; F_{2}\right)+\bar{N}_{0}\left(r, \infty ; F_{3}\right) & =N_{2}^{0}(r, \infty ; H(G-1))+N_{0}(r, \infty ; H) \\
& \leq N_{2}^{0}(r, \infty ; F)+\bar{N}_{0}(r, \infty ; F) \leq 3 \bar{N}_{0}(r, \infty ; F), \\
N_{2}^{0}\left(r, \infty ; F_{3}\right)+\bar{N}_{0}\left(r, \infty ; F_{1}\right) & =N_{2}^{0}(r, \infty ; H)+\bar{N}_{2}^{0}(r, \infty ; F) \\
& \leq 2 \bar{N}_{0}(r, \infty ; H)+\bar{N}_{0}(r, \infty ; F) \leq 3 \bar{N}_{0}(r, \infty ; F)
\end{aligned}
$$


and similarly for the other three terms. So from (8) we get

$$
\begin{aligned}
T_{0}(r, F) \leq & N_{0}(r, 0 ; F)+N_{0}(r, 0 ; G)+3 \bar{N}_{0}(r, \infty ; F) \\
& +4 \bar{N}_{0}(r, \infty ; G)+o\left(\max \left\{T_{0}(r, F), T_{0}(r, G)\right\}\right) .
\end{aligned}
$$

Now we put $G_{1}=-F_{2} / F_{3}=G, G_{2}=1 / F_{3}=1 / H$ and $G_{3}=-F_{1} / F_{3}=$ $-F / H$. Then by Lemma $6, G_{1}, G_{2}, G_{3}$ are linearly independent and so proceeding as above we get

$$
\begin{aligned}
T_{0}(r, G) \leq & N_{0}(r, 0 ; F)+N_{0}(r, 0 ; G)+3 \bar{N}_{0}(r, \infty ; G) \\
& +4 \bar{N}_{0}(r, \infty ; F)+o\left(\max \left\{T_{0}(r, F), T_{0}(r, G)\right\}\right) .
\end{aligned}
$$

From (9) and (10) we get

$$
\begin{aligned}
\max \left\{T_{0}(r, F), T_{0}(r, G)\right\} \leq & \left(10-\delta_{0}(0 ; F)-\delta_{0}(0 ; G)-4 \Theta_{0}(\infty ; F)\right. \\
& \left.-4 \Theta_{0}(\infty ; G)+o(1)\right) \max \left\{T_{0}(r, F), T_{0}(r, G)\right\} \\
< & (1-\varepsilon+o(1)) \max \left\{T_{0}(r, F), T_{0}(r, G)\right\},
\end{aligned}
$$

which is a contradiction, where by (5) we choose

$$
0<\varepsilon<\delta_{0}(0 ; F)+\delta_{0}(0 ; G)+4 \Theta_{0}(\infty ; F)+4 \Theta_{0}(\infty ; G)-9 .
$$

Hence there exist constants $c_{1}, c_{2}, c_{3}$, not all zero, such that

$$
c_{1} F_{1}+c_{2} F_{2}+c_{3} F_{3} \equiv 0 .
$$

Clearly $c_{1} \neq 0$. For, otherwise from (11) we get $H\left(c_{3}-c_{2} G\right) \equiv 0$, which is impossible because $F$ and $G$ are nonconstant.

Now eliminating $F_{1}$ from (7) and (11) we get

$$
c F_{2}+d F_{3} \equiv 1
$$

where $c=1-c_{2} / c_{1}$ and $d=1-c_{3} / c_{1}$.

If possible let $c d \neq 0$. Then from $(12)$ we get $(c / d)(G)+1 /(d H) \equiv 1$. So by Lemma 3 we get

$$
T_{0}(r, G) \leq N_{0}(r, 0 ; G)+\bar{N}_{0}(r, \infty ; H)+\bar{N}_{0}(r, \infty ; G)+S_{0}(r, G),
$$

i.e.

$$
T_{0}(r, G) \leq N_{0}(r, 0 ; G)+\bar{N}_{0}(r, \infty ; F)+\bar{N}_{0}(r, \infty ; G)+S_{0}(r, G) .
$$

By the second fundamental theorem we get on integration

$$
\begin{aligned}
T_{0}(r, F) & \leq N_{0}(r, 0 ; F)+N_{0}(r, 1 ; F)+\bar{N}_{0}(r, \infty ; F)+S_{0}(r ; F) \\
& =N_{0}(r, 0 ; F)+N_{0}(r, 1 ; G)+\bar{N}_{0}(r, \infty ; F)+S_{0}(r, F) \\
& \leq N_{0}(r, 0 ; F)+T_{0}(r, G)+\bar{N}_{0}(r, \infty ; F)+S_{0}(r, F) .
\end{aligned}
$$

So by (13) we obtain

$$
\begin{aligned}
T_{0}(r, F) \leq & N_{0}(r, 0 ; F)+N_{0}(r, 0 ; G)+2 \bar{N}_{0}(r, \infty ; F) \\
& +\bar{N}_{0}(r, \infty ; G)+S_{0}(r, F)+S_{0}(r, G) .
\end{aligned}
$$


From (13) and (14) we get

$$
\begin{aligned}
\max \left\{T_{0}(r, F), T_{0}(r, G)\right\} \leq & N_{0}(r, 0 ; F)+N_{0}(r, 0 ; G)+2 \bar{N}_{0}(r, \infty ; F) \\
& +\bar{N}_{0}(r, \infty ; G)+o\left(\max \left\{T_{0}(r, F), T_{0}(r, G)\right\}\right)
\end{aligned}
$$

and so $\delta_{0}(0 ; F)+\delta_{0}(0 ; G)+2 \Theta_{0}(\infty ; F)+\Theta_{0}(\infty ; G) \leq 4$.

Now by $(5)$ we see that

$$
\begin{aligned}
9 & <\delta_{0}(0 ; F)+\delta_{0}(0 ; G)+4 \Theta_{0}(\infty ; F)+4 \Theta_{0}(\infty ; G) \\
& \leq 4+2 \Theta_{0}(\infty ; F)+3 \Theta_{0}(\infty ; G) \leq 9,
\end{aligned}
$$

which is a contradiction. Therefore $c d=0$. From (12) we see that $c$ and $d$ are not simultaneously zero. So we consider the following cases.

CASE I. Let $d=0$. Then from (12) we get $-c F+1 / G \equiv 1-c$. If $c \neq 1$, we obtain $(-c /(1-c)) F+1 /((1-c) G) \equiv 1$. So by Lemma 3 it follows that

$$
T_{0}(r, F) \leq N_{0}(r, 0 ; F)+\bar{N}_{0}(r, \infty ; G)+\bar{N}_{0}(r, \infty ; F)+S_{0}(r, F)
$$

and

$$
\begin{aligned}
T_{0}(r, G) & =T_{0}(r, 1 / G)+S_{0}(r, G) \\
& \leq N_{0}(r, 0 ; F)+N_{0}(r, 0 ; G)+\bar{N}_{0}(r, \infty ; G)+S_{0}(r, G) .
\end{aligned}
$$

Hence

$$
\begin{aligned}
\max \left\{T_{0}(r, F), T_{0}(r, G)\right\} \leq & N_{0}(r, 0 ; F)+N_{0}(r, 0 ; G)+\bar{N}_{0}(r, \infty ; F) \\
& +\bar{N}_{0}(r, \infty ; G)+o\left(\max \left\{T_{0}(r, F), T_{0}(r, G)\right\}\right),
\end{aligned}
$$

and so $\delta_{0}(0 ; F)+\delta_{0}(0 ; G)+\Theta_{0}(\infty ; F)+\Theta_{0}(\infty ; G) \leq 3$.

From (5) we see that

$$
\begin{aligned}
9 & <\delta_{0}(0 ; F)+\delta_{0}(0 ; G)+4 \Theta_{0}(\infty ; F)+4 \Theta_{0}(\infty ; G) \\
& \leq 3+3 \Theta_{0}(\infty ; F)+3 \Theta_{0}(\infty ; G) \leq 9,
\end{aligned}
$$

which is a contradiction. Therefore $c=1$ and so $F G \equiv 1$, i.e., $[\Psi(D) f]$. $[\Psi(D) g] \equiv 1$.

CASE II. Let $c=0$. Then from (12) we get $d F-G \equiv d-1$. If $d \neq 1$ it follows that $(d /(d-1)) F-(1 /(d-1)) G \equiv 1$. Now by Lemma 3 we obtain

$$
\begin{aligned}
& T_{0}(r, F) \leq N_{0}(r, 0 ; F)+N_{0}(r, 0 ; G)+\bar{N}_{0}(r, \infty ; F)+S_{0}(r, F), \\
& T_{0}(r, G) \leq N_{0}(r, 0 ; F)+N_{0}(r, 0 ; G)+\bar{N}_{0}(r, \infty ; G)+S_{0}(r, G) .
\end{aligned}
$$

So we get

$$
\begin{aligned}
\max \left\{T_{0}(r, F), T_{0}(r, G)\right\} \leq & N_{0}(r, 0 ; F)+N_{0}(r, 0 ; G)+\bar{N}_{0}(r, \infty ; F) \\
& +\bar{N}_{0}(r, \infty ; G)+o\left(\max \left\{T_{0}(r, F), T_{0}(r, G)\right\}\right)
\end{aligned}
$$

and as in Case I this leads to a contradiction. So $d=1$ and hence $F \equiv G$, i.e., $\Psi(D)(f-g) \equiv 0$. Therefore $f-g \equiv s$ where $s=s(z)$ is a solution of $\Psi(D) w=0$. This proves the theorem. 
Proof of Theorem 2. If $f$ and $g$ are of finite order, we can prove along the lines of Lemmas 5 and 8 that

$$
\delta(0 ; \Psi(D) f) \geq \frac{\sum_{a \neq \infty} \delta(a ; f)}{1+p(1-\Theta(\infty ; f))}, \quad \Theta(\infty ; \Psi(D) f) \geq 1-\frac{1-\Theta(\infty ; f)}{\sum_{a \neq \infty} \delta_{p}(a ; f)},
$$

and the corresponding results for $g$. Let $F=\Psi(D) f$ and $G=\Psi(D) g$. Then by the condition (ii) of the theorem we get

$$
\delta(0 ; F)+\delta(0 ; G)+2 \Theta(\infty ; F)+2 \Theta(\infty ; G)>5 .
$$

This implies

$$
\begin{aligned}
\limsup _{r \rightarrow \infty} \frac{N(r, 0 ; F)}{T(r, F)} & +\limsup _{r \rightarrow \infty} \frac{N(r, 0 ; G)}{T(r, G)} \\
& +2 \limsup _{r \rightarrow \infty} \frac{\bar{N}(r, \infty ; F)}{T(r, F)}+2 \limsup _{r \rightarrow \infty} \frac{\bar{N}(r, \infty ; G)}{T(r, G)}<1,
\end{aligned}
$$

i.e.,

$$
\limsup _{r \rightarrow \infty} \frac{N_{2}(r, 0 ; F)+N_{2}(r, 0 ; G)+N_{2}(r, \infty ; F)+N_{2}(r, \infty ; G)}{\max \{T(r, F), T(r, G)\}}<1
$$

and so by Lemma 9 the theorem follows.

Considering $f=-2^{-n} e^{2 z}+e^{z}, g(z)=-(-1)^{n} 2^{-n} e^{-2 z}+(-1)^{n} e^{-z}$ where $n$ is a positive integer, Yi and Yang [16] claimed that for $n \geq 1$ the condition $\delta(0 ; f)+\delta(0 ; g)>1$ of Theorem $\mathrm{G}$ is necessary. In the following example we see that this claim is not justified.

EXAmPle 2. Let $f=e^{z}-1$ and $g=1+(-1)^{n} e^{-z}$. Then $\delta(0 ; f)=$ $\delta(0 ; g)=0$ and $f^{(n)}, g^{(n)}$ share 1 CM. Also $f^{(n)} g^{(n)} \equiv 1$.

In the first corollary we improve Theorem $\mathrm{G}$ for $n \geq 1$.

Corollary 1. Let $f, g$ be two meromorphic function with $\Theta(\infty ; f)=$ $\Theta(\infty ; g)=1$. If for $n \geq 1$ the derivatives $f^{(n)}$ and $g^{(n)}$ are nonconstant and share 1 CM with

(i) $\sum_{a \neq \infty} \delta(a ; f)+\sum_{a \neq \infty} \delta(a ; g)>1$,

(ii) $\Theta(0 ; f)+\Theta(0 ; g)>1$,

then either (a) $f^{(n)} g^{(n)} \equiv 1$ or (b) $f \equiv g$.

Proof. Choosing $\Psi(D)=D^{n}$, from Theorem 1 it follows that either $f^{(n)} g^{(n)} \equiv 1$ or $f-g \equiv Q$, where $Q$ is a polynomial of degree at most $n-1$. If possible, let $Q \not \equiv 0$. Then from [3, Theorem 2.5, p. 47] it follows that

$$
\begin{aligned}
T(r, f) & \leq \bar{N}(r, 0 ; f)+\bar{N}(r, Q ; f)+\bar{N}(r, \infty ; f)+S(r, f) \\
& =\bar{N}(r, 0 ; f)+\bar{N}(r, 0 ; g)+\bar{N}(r, \infty ; f)+S(r, f) .
\end{aligned}
$$


Since $f-g \equiv Q$, it follows that $T(r, f)=T(r, g)+O(\log r)$. So we get $\Theta(0 ; f)+\Theta(0 ; g) \leq 1$, which is a contradiction. Therefore $Q \equiv 0$ and so $f \equiv g$.

The following examples show that the condition $\Theta(0 ; f)+\Theta(0 ; g)>1$ is necessary for the validity of case (b).

ExAmPle 3. Let $f=e^{z}+1$ and $g=e^{z}$. Then $\Theta(0 ; f)=0, \Theta(0 ; g)=1$, $\sum_{a \neq \infty} \delta(a ; f)+\sum_{a \neq \infty} \delta(a ; g)=2, \Theta(\infty ; f)=\Theta(\infty ; g)=1$ and $f^{(n)}, g^{(n)}$ share 1 CM but $f-g \equiv 1$.

ExAmple 4. Let $f=e^{z}+1$ and $g=(-1)^{n} e^{-z}$. Then $\Theta(0 ; f)=0$, $\Theta(0 ; g)=1, \sum_{a \neq \infty} \delta(a ; f)+\sum_{a \neq \infty} \delta(a ; g)=2, \Theta(\infty ; f)=\Theta(\infty ; g)=1$ and $f^{(n)}, g^{(n)}$ share 1 CM but $f^{(n)} g^{(n)} \equiv 1$.

Answering the question of C. C. Yang [9], mentioned in the introduction, H. X. Yi [12] proved the following theorem.

TheOREm H [12]. Let $f$ and $g$ be two nonconstant entire functions. Assume that $f, g$ share $0 C M$ and $f^{(n)}, g^{(n)}$ share $1 C M$, where $n$ is a nonnegative integer. If $\delta(0 ; f)>1 / 2$ then either $f \equiv g$ or $f^{(n)} g^{(n)} \equiv 1$.

Considering $f=-2^{-n} e^{2 z}+(-1)^{n+1} 2^{-n} e^{z}$ and $g=(-1)^{n+1} 2^{-n} e^{-2 z}-$ $2^{-n} e^{-z}$, Yi [12] claimed that the condition $\delta(0 ; f)>1 / 2$ is necessary. The following example shows that for $n \geq 1$ this is not always the case.

EXAmple 5. Let $f=e^{z}-1$ nad $g=(-1)^{n+1}+(-1)^{n} e^{-z}$. Then $f, g$ share $0 \mathrm{CM}$ and $f^{(n)}, g^{(n)}(n \geq 1)$ share $1 \mathrm{CM}, \delta(0 ; f)=0$ but $f^{(n)} g^{(n)} \equiv 1$.

In the following corollary we provide an answer to a question of Yang [9].

Corollary 2. Let $f$ and $g$ be two meromorphic functions with $\Theta(\infty ; f)$ $=\Theta(\infty ; g)=1$. Suppose that $f^{(n)}, g^{(n)}(n \geq 1)$ share $1 C M$ and $f, g$ share a value $b(\neq \infty)$ IM. If $\sum_{a \neq \infty} \delta(a ; f)+\sum_{a \neq \infty} \delta(a ; g)>1$ then either $f \equiv g$ or $f^{(n)} g^{(n)} \equiv 1$.

Proof. The condition $\sum_{a \neq \infty} \delta(a ; f)+\sum_{a \neq \infty} \delta(a ; g)>1$ implies that $f$ and $g$ are transcendental so that $f^{(n)}, g^{(n)}$ are nonconstant. Choosing $\Psi(D)=D^{n}$ we see from Theorem 1 that either $f^{(n)} g^{(n)} \equiv 1$ or $f-g \equiv Q$, where $Q$ is a polynomial. Now we consider the case $f-g \equiv Q$. If possible, let $Q \not \equiv 0$. Also we suppose that $f$ has at most a finite number of $b$-points and so $g$ also has a finite number of $b$-points. Now by [3, Theorem 2.5, p. 47] it follows that

$$
\begin{aligned}
T(r, f) & \leq \bar{N}(r, b ; f)+\bar{N}(r, b+Q ; f)+\bar{N}(r, \infty ; f)+S(r, f) \\
& =\bar{N}(r, b ; f)+\bar{N}(r, b ; g)+S(r, f)=O(\log r)+S(r, f),
\end{aligned}
$$

which is a contradiction. Therefore $f$ has infinitely many $b$-points and so $f-g$ has infinitely many zeros. This again implies a contradiction because $f-g \equiv Q$ and $Q \not \equiv 0$. So $Q \equiv 0$ and hence $f \equiv g$. 
Considering $f=-2^{-n} e^{2 z}+(-1)^{n+1} 2^{-n} e^{z}$ and $g=(-1)^{n+1} 2^{-n} e^{-2 z}-$ $2^{-n} e^{-z}$ we can verify that the condition $\sum_{a \neq \infty} \delta(a ; f)+\sum_{a \neq \infty} \delta(a ; g)>1$ of Corollary 2 is necessary.

Corollary 3. Let $\Psi(D)=D\left(D-\lambda_{1}\right)\left(D-\lambda_{2}\right) \ldots\left(D-\lambda_{p-1}\right)$ where $\lambda_{i}$ 's are nonzero pairwise distinct complex numbers. Also suppose that $f$ and $g$ are two meromorphic functions with the following properties:

(i) $\Psi(D) f, \Psi(D) g$ are nonconstant and share $1 C M$,

(ii) $\sum_{a \neq \infty} \delta(a ; f)+\sum_{a \neq \infty} \delta(a ; g)>1$ and $\Theta(\infty ; f)=\Theta(\infty ; g)=1$,

(iii) $f$ and $g$ have $b$-points $(b \neq \infty)$ with multiplicities not less than $p+1$ at the origin.

Then $f \equiv g$.

Proof. From the theorem we get either $[\Psi(D) f] \cdot[\Psi(D) g] \equiv 1$ or $f-g \equiv$ $c_{0}+c_{1} e^{\lambda_{1} z}+c_{2} e^{\lambda_{2} z}+\ldots+c_{p-1} e^{\lambda_{p-1} z}$ where $c_{i}$ 's are constants. Since $f$ has a $b$-point with multiplicity at least $p+1$ at the origin, it follows that $\Psi(D) f$ has at least a simple zero at the origin. Similarly $\Psi(D) g$ has at least a simple zero at the origin. So the case $[\Psi(D) f] \cdot[\Psi(D) g] \equiv 1$ does not occur. If possible, let $f \not \equiv g$. Then the constants $c_{0}, c_{1}, \ldots, c_{p-1}$ are not all zero. Also by condition (iii) it follows that $f-g$ has a zero at the origin with multiplicity at least $p+1$. This implies that

$$
\sum_{i=0}^{p-1} c_{i}=0, \quad \sum_{i=0}^{p-1} \lambda_{i} c_{i}=0, \quad \sum_{i=0}^{p-1} \lambda_{i}^{2} c_{i}=0, \ldots, \sum_{i=0}^{p-1} \lambda_{i}^{p} c_{i}=0 .
$$

This system of equations gives $c_{0}=c_{1}=c_{2}=\ldots=c_{p-1}=0$, which is a contradiction. Therefore $f \equiv g$. This proves the corollary.

The following examples show that condition (iii) of Corollary 3 is necessary.

EXAMPle 6 . Let $f=e^{z^{3}}, g=e^{z^{3}}+1$ and $\Psi(D)=D(D-1)$. Then $\Psi(D) f, \Psi(D) g$ share $1 \mathrm{CM}, \sum_{a \neq \infty} \delta(a ; f)+\sum_{a \neq \infty} \delta(a ; g)=2, \Theta(\infty ; f)=$ $\Theta(\infty ; g)=1$ and $f-1, g-2$ have zeros with multiplicity three at the origin, but $f \not \equiv g$.

EXAmple 7. Let $f=e^{z}-1, g=1-e^{-z}$ and $\Psi(D)=D$. Then $\Psi(D) f$, $\Psi(D) g$ share $1 \mathrm{CM}, \sum_{a \neq \infty} \delta(a ; f)+\sum_{a \neq \infty} \delta(a ; g)=2, \Theta(\infty ; f)=\Theta(\infty ; g)$ $=1$ and $f, g$ have simple zeros at the origin, but $f \not \equiv g$.

Let us conclude the paper with the following question: What can be said if two nonlinear differential polynomials generated by two meromorphic functions share $1 \mathrm{CM}$ ?

Acknowledgements. The author is grateful to the referee for his valuable suggestions. 


\section{References}

[1] M. Furuta and N. Toda, On exceptional values of meromorphic functions of divergence class, J. Math. Soc. Japan 25 (1973), 667-679.

[2] F. Gross, Factorization of Meromorphic Functions, U.S. Govt. Math. Res. Center, Washington, D.C., 1972.

[3] W. K. Hayman, Meromorphic Functions, The Clarendon Press, Oxford, 1964.

[4] I. Lahiri and D. K. Sharma, The characteristic function and exceptional value of the differential polynomial of a meromorphic function, Indian J. Pure Appl. Math. 24 (1993), 779-790.

[5] P. Li and C. C. Yang, Some further results on the unique range sets of meromorphic functions, Kodai Math. J. 18 (1995), 437-450.

[6] M. Ozawa, Unicity theorems for entire functions, J. Anal. Math. 30 (1976), 411420 .

[7] K. Shibazaki, Unicity theorems for entire functions of finite order, Mem. National Defense Acad. Japan 21 (1981), no. 3, 67-71.

[8] N. Toda, On a modified deficiency of meromorphic functions, Tôhoku Math. J. 22 (1970), 635-658.

[9] C. C. Yang, On two entire functions which together with their first derivatives have the same zeros, J. Math. Anal. Appl. 56 (1976), 1-6.

[10] H. X. Yi, Meromorphic functions with two deficient values, Acta Math. Sinica 30 (1987), 588-597.

[11] - Meromorphic functions that share two or three values, Kodai Math. J. 13 (1990), 363-372.

[12] - A question of C. C. Yang on the uniqueness of entire functions, ibid. 13 (1990), $39-46$.

[13] - Unicity theorems for entire or meromorphic functions, Acta Math. Sinica (N.S.) 10 (1994), 121-131.

[14] - Meromorphic functions that share one or two values, Complex Variables Theory Appl. 28 (1995), 1-11.

[15] H. X. Yi and C. C. Yang, Unicity theorems for two meromorphic functions with their first derivatives having the same 1-points, Acta Math. Sinica 34 (1991), 675680 .

[16] —, - A uniqueness theorem for meromorphic functions whose nth derivative share the same 1-points, J. Anal. Math. 62 (1994), 261-270.

Department of Mathematics

Jadavpur University

Calcutta 700032, India
Present address:

Department of Mathematics

University of Kalyani

Kalyani 741235

West Bengal, India

E-mail: indrajit@cal2.vsnl.net.in 